\title{
Letter to the Editor: Some thoughts concerning dealing with an aneurysmal disease in children with Takayasu arteritis: a rare but aggressive vasculitis
}

Keywords: Aneurysmal disease in children; diagnostic tools; PET-CT; Takayasu; aneurysm

Received: 22 April 2016; Accepted: 19 June 2016; First published online: 18 October 2016

We acknowledge the University of Michigan's surgical department members for their recent publication on Takayasu disease. ${ }^{1}$ We found the case of a 10 -year-old boy very interesting as it shows the extremes of the disease and its management. The young age at initial presentation (3 years), the poor response to the dual therapy, the appearance of an aneurysm, and its enormous diameter certainly fall beyond the scope of a typical presentation. The hindrances of surgical management are also remarkable, and the determination to carry on deserves appreciation. We would like to know whether, based on the authors' best judgement, extending the scope of the procedure would have any potential to reduce the risk of secondary aneurysm formation.

On the basis of our own experience, the timing, type, and extent of the surgical procedure are the biggest concerns in the management of a complicated Takayasu case. Recently, a 17-year-old male with a giant aneurysm in the ascending aorta and brachiocephalic trunk with accompanying arterial wall thickening found on CT scan uneventfully underwent the Yacoub procedure with ascending aorta, proximal arch, and proximal brachiocephalic trunk replacement at our institution. The patient continued to present clinical and laboratory symptoms of the disease. We have chosen positron emission tomography - computed tomography (PET/ $\mathrm{CT}$ ) as a follow-up modality for its advantage of adding functional data to the anatomical imaging. ${ }^{2}$ The PET/CT clearly showed the active sites of inflammation within the main pulmonary artery, distal arch, distal brachiocephalic trunk, and jugular arteries.
PET-CT is a modality of limited availability, is expensive, and most importantly it exposes the patient to approximately double dose of radiation, ${ }^{3}$ but the gain may be worthwhile. We believe that this modality may prove useful during initial diagnosis as a roadmap for further Doppler follow-up; however, whether it has a potential to prove its usefulness in planning the extent of surgical interventions remains an open question. Owing to a very low incidence of the disease, it will be hard to determine the superiority of one approach over the other, but every approach leading to improvement in the management of this chronic disease is worth considering.

$$
\begin{array}{r}
\text { Joanna Kwiatkowska, Jarostaw Meyer-Szary } \\
\text { Department of Pediatric Cardiology and Congenital } \\
\text { Heart Disease, } \\
\text { Medical University of Gdansk, Gdansk, Poland } \\
\text { Rafat Pawlaczyk } \\
\text { Department of Cardiovascular Surgery, Medical } \\
\text { University of Gdansk, Gdansk, Poland }
\end{array}
$$

\section{References}

1. Halaweish I, Patel H, Si M-S. Giant aortic aneurysm in a child with Takayasu arteritis. Cardiol Young 2016; 26: 593-595.

2. Tezuka D, Haraguchi G, Ishihara T, et al. Role of FDG PET-CT in Takayasu arteritis: sensitive detection of recurrences. JACC Cardiovasc Imaging 2012; 5: 422-429.

3. Huang B, Law MW-M, Khong P-L. Whole-body PET/CT scanning: estimation of radiation dose and cancer risk. Radiology 2009; 251: 166-174. 